\title{
Single-cell epigenetics
}

Measuring DNA methylation at specific sites in single cells sheds light on early embryo development.

It is hard enough to understand how a mutated gene causes a particular defect in an organism; all bets are off when the defect changes from individual to individual. Daniel Messerschmidt and William Burkholder at the Agency for Science, Technology and Research in Singapore and their fellow researchers faced this problem when studying genetically identical mouse mutant embryos. "We were seeing these very bizarre phenotypes ... they were heterogeneous, all over the place," says Messerschmidt. Tracing the source of the mysterious diversity led them to develop a way to study DNA methylation, a heritable epigenetic modification that regulates gene activity, in single cells of the very early embryo.

Life begins when the genetic contributions of the parents meet in the fertilized egg. For the new combination to work, the epigenetic baggage that accumulated in the parents' lifetime must be cast offwith important exceptions. Certain genes are imprinted, meaning that only the gene copy inherited from one parent will be expressed in healthy offspring. This favoritism is associated with DNA methylation of the silent copy, which a group of proteins are dispatched to selectively protect during epigenetic reprogramming.

The mutants that the researchers studied were defective in Trim28, one of the genes required for imprinting maintenance in the mouse. A mutation in the mother could affect her genetically normal pups, "meaning that the effect should only happen very early on, in the first few days, when there is no embryonic gene transcription," says Messerschmidt. The researchers hypothesized that phenotypic variability in older embryos could be due to the patchy effects of lost imprinting mediated by the maternal mutation in the early embryo. To prove it, they needed to examine individual cells.

Single-cell epigenetics is new territory. Messerschmidt notes that most methods need DNA from a large number of cells as starting material for efficient immunoprecipitation or in order to buffer losses during the chemical conversion used for sequencing methylated bases.
Instead, the researchers chose to scale down a methylation assay that targets specific sites in the genome. The assay uses a restriction enzyme that can cut only unmethylated DNA and thereby distinguish it from methylated DNA in a subsequent PCR amplification step. "It has been done in single cells before, but the efficiency was very, very low, so you had to analyze a lot of single cells," says Messerschmidt. Moreover, it was usually only possible to analyze one gene per cell. The team's new approach hinges on a commercial microfluidic platform, which allows the genetic material from a single cell to be evenly distributed into nanoliter volumes. Their modifications boosted efficiency to a near-perfect success rate, and they could check methylation in six genes simultaneously in one cell.

Applying the method to lone cells from dissociated eight-cell-stage embryos revealed variable imprinting in the six genes in Trim 28 mutants. The patchy nature of the effects is likely due to an uneven reduction in methylation in the early embryo that, in some cells, dips too low for the functioning paternal Trim 28 copy to maintain or 'protect' through future cell divisions.

The team is broadening the technology. They are working on conditions to test every known imprinted gene in mouse and human and can already analyze more than six genes simultaneously from the same cell. Mature tissues such as tumor tissue or stem cell niches are also in sight, says Messerschmidt. These can be dissociated and sorted to capture single cells, which can be processed to look at heterogeneity in DNA methylation.

Messerschmidt is also collaborating with clinicians to develop assays to screen in vitro-fertilized (IVF) embryos as another promising application of single-cell epigenetics. Increasing evidence shows that culture methods may introduce epigenetic variability in IVF embryos, which may drop the procedure's success rates, he says. Singlecell epigenetic approaches will be critical for understanding heterogeneity in development and disease.

Tal Nawy

\section{RESEARCH PAPERS}

Lorthongpanich, C. et al. Single-cell DNA-methylation analysis reveals epigenetic chimerism in preimplantation embryos. Science 341, 1110-1112 (2013). 